\title{
Norois
}

Environnement, aménagement, société

\section{Sentir et ressentir Paris. L'exemple du quai du RER B à Châtelet-les Halles}

\section{Lucile Grésillon}

\section{(2) OpenEdition \\ 1 Journals}

Édition électronique

URL : https://journals.openedition.org/norois/4637

DOI : 10.4000/norois. 4637

ISBN : 978-2-7535-2916-8

ISSN : $1760-8546$

\section{Éditeur}

Presses universitaires de Rennes

\section{Édition imprimée}

Date de publication : 30 juin 2013

Pagination : 11-24

ISBN : 978-2-7535-2882-6

ISSN : 0029-182X

\section{Référence électronique}




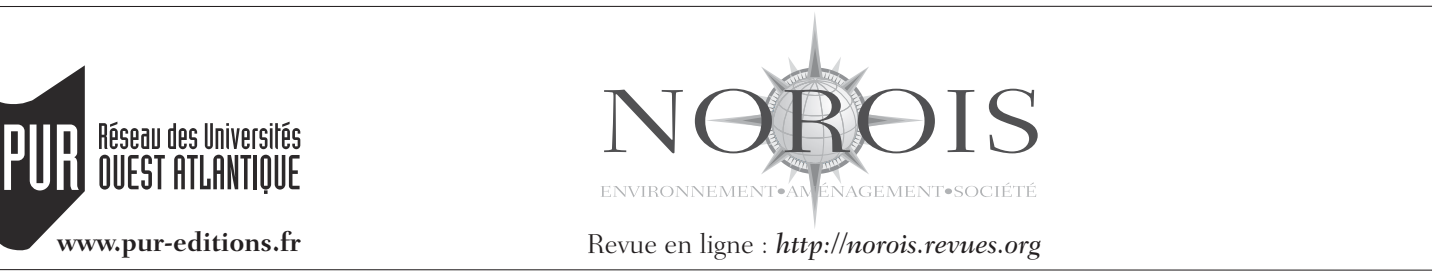

\title{
Sentir et ressentir Paris L'exemple du quai du RER B à Châtelet-les Halles
}

\author{
Sensing and Feeling Paris: Metro Station "Châtelet-les Halles" Exemple
}

\author{
Lucile GrÉSILLON
}

Maître de conférences en géographie, Université de Caen - UMR ESO, Maison de la Recherche en Sciences
Humaines, Esplanade de la Paix - 14032 CAEn cedex (lucilegresillon@gmail.com)

Résumé : Comment prendre en compte notre expérience sensible des milieux urbains ? Comment expliquer la difficulté en géographie de rendre compte de ce qui est senti olfactivement et ressenti dans les lieux? En réponse à ces questions, cet article propose de mettre en regard l'exercice traditionnel de description en géographie, les méthodes géographiques d'investigation de terrain et les savoirs des neurosciences - en particulier de la neurophysiologie de l'olfaction - et d'en extraire des apports possibles. Cet exposé se fonde sur une recherche exploratoire de doctorat en géographie (Grésillon, 2005, 2010b) sur les odeurs dans cinq lieux parisiens dont la station Châtelet-les Halles. Nous commencerons cet article par poser les fondements géographiques et philosophiques, d'en présenter les limites et la contribution que peuvent y apporter les neurosciences. Nous continuerons par une description exhaustive du lieu d'investigation d'après des recherches documentaires, des rencontres d'acteurs et les ressentis de la chercheure. Nous exposerons ensuite rapidement la méthode d'enquête faite de questionnaires et d'analyse textuelle, les odeurs perçues et les ressentis observés. Nous clôturerons par la compréhension de l'exercice de la description que cette étude permet et par les questions soulevées.

\begin{abstract}
How can we take into account our sensitive experience of urban areas? How can we explain the difficulty in geography to report what is "olfactively" experienced and felt in different locations? The purpose of this article is to face those questions, comparing conventional descriptive method in geography with field investigation and knowledge of neurosciences - especially regarding olfactory neurophysiology - in order to extract relevant information. This paper is based on a doctoral exploratory research (Grésillon, 2005, 2010b) regarding odours of five different places in Paris, including metro station "Châtelet-les Halles". We will start by exposing geographical and philosophical basis, showing their limits and how neurosciences could contribute to overcome these. We will follow with a complete description of the different urban sites where the investigation takes place, including documentary research, meetings with actors, and feelings of the researcher. We will then continue with an explanation of the methods used, based on questionnaires, textual analysis, odours perceived and feelings observed. And finally, we will conclude with a discussion of the descriptive exercise that this study allowed, and the questions it raises.
\end{abstract}

Mots clés : Experience sensible - olfaction - Paris - méthode - odeurs

Keywords: Sensitive experience - olfaction - Paris - méthod - odors 


\section{INTRODUCTION}

Comment prendre en compte notre expérience sensible des lieux? Comment rendre compte du sentir et du ressentir dans notre relation au milieu urbain? À partir de ces questions, présentes en géographie depuis une vingtaine d'années (cf. article de Th. Manola dans ce numéro), cet article propose de mettre en regard les méthodes géographiques d'investigation de terrain et les savoirs des neurosciences - en particulier de la neurophysiologie de l'olfaction - et d'en extraire des apports possibles. Le caractère polysémique du verbe «sentir » figurant dans le titre offre cette possibilité. Au sens concret, il signifie l'action de percevoir olfactivement ou tactilement un objet ou une personne. Au sens abstrait, il insiste sur le fait de percevoir une action, quelque chose ou quelqu'un ( «je le sens mal») ou d'être dans un état de bien-être («je me sens bien»). Son usage peut aller jusqu'à formuler le fait de ne pas aimer une personne, comme dans l'expression : « je ne peux pas le sentir». Sentir, ne pas sentir, ne pas pouvoir sentir un lieu comme un espace de transport en commun parisien serait donc une question de sensorialité mais également de sensibilité. L'usage du terme ressentir quant à lui insiste sur l'émotion, le plaisir et le déplaisir éprouvés à parcourir et corrélativement à sentir un endroit de Paris. Il s'agit alors, dans cet écrit, de présenter les interrogations fondées, à l'origine, sur une insatisfaction personnelle face aux rendus géographiques de l'expérience sensible des lieux. Ces questionnements ont donné lieu à une recherche exploratoire de doctorat en géographie sur les odeurs dans cinq lieux parisiens dont la station Châtelet-Les Halles. Ce travail - effectué entre 1999 et 2005 - a été publié après des remaniements et des enrichissements en 2010 (Grésillon, 2010b). L'attrait des odeurs et du sens olfactif repose sur un double constat : une acuité olfactive importante de la chercheure ainsi qu'une pauvreté des travaux en géographie sur le sujet. Cet état de fait avait déjà été l'objet d'un mémoire de maitrise (Grésillon, 1997) et d'un chapitre dans un ouvrage (Grésillon, 1998). Le dessein de cet article est également d'exposer les pistes de compréhension proposées par les neurosciences.

En quoi une enquête sur ce qui est senti olfactivement et ressenti par les voyageurs sur le quai de RER B à Châtelet-Les Halles, mise en regard avec les recherches documentaires et le vécu de la chercheure, peut-elle nous éclairer sur la difficulté de rendre compte scientifiquement du rapport sensible à l'espace observé dans l'exercice de description chère à la géographie?

Nous commencerons cet article en posant les fondements géographiques et philosophiques de cette problématique de la restitution de l'expérience olfactive des lieux, en présentant les limites des approches classique et la contribution que peuvent y apporter les neurosciences. Nous poursuivrons par une description exhaustive du lieu d'investigation à partir des recherches documentaires, des rencontres d'acteurs et des ressentis de la chercheure. Nous exposerons ensuite rapidement - afin de donner un aperçu global - la méthode d'enquête faite de questionnaires et d'analyse textuelle, mais aussi les odeurs perçues et les ressentis observés ${ }^{1}$. Nous terminerons par l'évaluation de la portée de cette étude pour notre problématique et par les questions qu'elle soulève.

\section{SENTIR ET RESSENTIR EN GÉOGRAPHIE, EN PHÉNOMÉNOLOGIE ET EN NEUROSCIENCES}

Ce n'est que récemment que le fait de sentir et de ressentir dans les lieux, autrement dit la dimension sensorielle et émotionnelle de notre rapport à l'espace se trouve frontalement pris en compte dans les recherches géographiques. Elle existe cependant, en filigrane, dès l'institutionnalisation de la géographie dans les écrits de Vidal de la Blache à travers l'exercice scientifique de la description mais reste cantonnée au visuel. Dans sa préface de l'Atlas Général d'histoire et de géographie (1895), qui deviendra le manifeste de l'École Française de Géographie, Vidal de la Blache présente ainsi son travail :

" J'ai cherché dans ce recueil à réunir sur chaque contrée l'ensemble des indications nécessaires pour obtenir une vue raisonnée. [...] Cette espèce de dossier [...] a pour but de placer sous les yeux l'ensemble des traits qui caractérisent une contrée, afin de permettre à l'esprit d'établir entre eux une liaison. »

\footnotetext{
1. Pour plus de détails sur ce sujet, nous invitons le lecteur à lire un autre article de l'auteure (Grésillon, 2010a).
} 
Voici comment ce même auteur présente la Seine à Paris dans son Tableau de la géographie de la France, non sans y inclure des marqueurs d'évaluation sensorielle (Vidal de la Blache, [1908], 1994, p. 216):

«Bien ouvert par son orientation aux rayons du soleil, dont les premiers feux l'éclairent et dont les feux couchant illuminent un des plus merveilleux panoramas urbains qu'on puisse voir, le fleuve trace à travers la ville un grand courant d'air et de lumière. »

La description du cours d'eau, qui est de l'ordre de la vue, renvoie au sentir, l'émerveillement décrit ramène au ressentir. Nous pouvons remarquer que le sensible reste cantonné au visuel. Toutefois, lors de l'avènement de l'École de la Géographie Française dans les années Trente, il n'existe plus aucune appréciation dans la description, le ressentir disparait. En 1934, Sion déplore l'appauvrissement de la description géographique depuis Vidal de la Blache : «N'avons-nous pas cependant péché par excès de prudence dans ce refus de mêler à notre travail de savant, notre vision et notre sentiment du pays? »(Sion, 1934). L'approche sensible des espaces à travers la description se trouve alors délaissée au profit d'une recherche d'objectivité dans la description favorisant l'explication : est-ce la solution trouvée pour asseoir la géographie dans les critères de scientificité des disciplines établies?

Contemporain de Vidal de la Blache, Reclus milite pour une géographie engagée et militante qui se démarque de celle du fondateur de la géographie institutionnelle. Elle intègre les relations symboliques, affectives et physiques des humains avec la terre. Dans la préface du tome 1 de L'Homme et la terre, Reclus évoque ([1905], 1982, p. 103) :

"L'émotion que l'on éprouve à contempler tous les paysages de la planète dans leur variété sans fin et dans l'harmonie que leur donne l'action des forces ethniques toujours en mouvement, cette même douceur des choses, on le ressent à voir la procession des hommes [...]. »

Ses descriptions se démarquent de celles de Vidal de La Blache puisqu'elles mobilisent la dimension olfactive (Reclus, tome 2, [1905], 1982) :
"Presque chaque agglomération urbaine est assombrie par un ou plusieurs faubourgs, hérissées de cheminées puantes, traversées par des rues noires $[\ldots]$. Que de villes $[\ldots]$ où l'air est presque irrespirable, où tout ce que l'on aperçoit, le sol, les routes, les murailles, le ciel, suintent la boue et le charbon!»

Cependant, cette prise en compte de notre relation charnelle à l'espace en géographie reste isolée. Il faut attendre l'impact du courant philosophique de la phénoménologie sur la géographie s'illustrant dans les écrits de Dardel, auteur d'un ouvrage intitulé lui aussi L'Homme et la terre (1952) pour que la sensibilité des êtres humains dans le rapport aux lieux s'affirme avec plus de légitimité. Les philosophes se réclamant de la phénoménologie déplacent les injonctions de Descartes en affirmant que, dans la perception, l'essentiel se trouve dans la relation entre le sujet et l'objet, dans ses modalités sensibles - émotionnelles, multisensorielles et hédoniques et non plus dans l'analyse des qualités de l'objet et du sujet. L'idée fondamentale se résume ainsi : dans la perception, le clivage sujet / objet est dépassé, il n'existe que des interactions entre propriétés physicochimiques $^{2}$ et sujets sentants. Pour reprendre la formulation de Merleau-Ponty, c'est « la vie ambiguë » (Merleau-Ponty, 1945), car «nous sommes mêlés au monde et aux autres dans une confusion inextricable » (Merleau-Ponty, 1945). MerleauPonty s'efforce de montrer que la perception est notre rapport originel au monde, " un type d'expérience originaire » (Merleau-Ponty, 1945). «Il ne faut donc pas se demander si nous percevons vraiment un monde, il faut dire au contraire : le monde est cela que nous percevons » (Merleau-Ponty, 1945). Le monde de la perception se découvre alors « comme berceau des significations, sens de tous les sens, et sol de toutes les pensées » (MerleauPonty, 1945). Dans cette lignée de pensée, Dardel préconise l'étude du «monde là où il est, rencontré d'abord à travers des sensations, des émotions, des sentiments, des croyances [...]». (Dardel, [1952],

2. L'ancienne catégorisation anatomique du système perceptif en cinq sens est remplacée en neurosciences aujourd'hui par une catégorie fonctionnelle liée à la nature des récepteurs : photorécepteurs, mécanorécepteurs, thermorécepteurs ou chimiorécepteurs. La vision et l'audition possèdent des capteurs physiques. L'olfaction et le goût sont régis par des chimiorécepteurs. La somesthésie (anciennement le toucher) est un sens à part car « hybride». Il possède des récepteurs physiques et chimiques. Du point de vue neurophysiologique, il n'existe donc pas cinq sens mais des récepteurs de nature différente, soit physique soit chimique. 
1990). Cependant ses écrits ne constituent pas formellement des descriptions sensibles de la surface terrestre, plutôt des interprétations généralisantes de sa propre expérience. Ces interprétations forment des supports à une démonstration idéologique et ne sont pas fondées sur l'étude de terrain. Cette philosophie continue d'imprégner la géographie par la suite. Frémont (1976) intègre les aspects subjectifs dans notre rapport au territoire grâce à la notion d'espace vécu. Berque poursuit la réflexion phénoménologique par la comparaison entre la relation à la nature en Europe et au Japon (1986). À la fin des années 1990 perdurent les analyses sur les rapports des êtres humains avec leurs lieux de vie à travers les concepts d'habiter (Hoyaux, 2002; Stock, 2004) et du mode d'habiter, développé au LADYSS par Mathieu et son groupe de travail $(1996,2004)$. Aujourd'hui, la relation affective des êtres humains à la ville se trouve tout particulièrement creusée par Martouzet $(2007,2010)$ et son équipe (Feildel, 2010) ainsi que, dans sa dimension politique, par Salomon-Cavin $(2005,2010)$.

En parallèle, à partir des années 1970, une autre lignée de la géographie se préoccupe de perception, dans le cadre de la géographie sociale, à l'intérieur d'un mouvement de recherche qui prend le nom de « géographie de la perception ». La définition de la représentation sur laquelle s'appuie ce courant provient des travaux développés par Piaget. Elle est la suivante : la représentation « consiste soit à évoquer des objets en leur absence, soit lorsqu'elle double la perception en leur présence, à compléter la connaissance perceptible en se référant à d'autres objets non actuellement perçus. » (Bailly, 1991). Bailly présente les premières réflexions dans un article intitulé "La perception des paysages urbains » (1974) paru dans l'Espace géographique. Il y reconnait la relativité de la perception de l'espace : « Le paysage, regardé comme un réseau de signifiés et de signifiants, est compris différemment par chacun qu'il soit individu ou groupe » (Bailly, 1991). Il introduit la notion de filtres qui limitent la perception du réel : «[...] l'information reçue de la réalité vécue résulte d'un filtrage opéré par les sens limités de l'homme [...]»(Bailly, 1991) décrivant en cela l'opération de sélection. Le deuxième filtre, celui qui provient de l'appréciation que l'on fait de la chose perçue et qui influence l'action, admis depuis moins longtemps, n'est pas évoqué. Cet axe de recherche fut ensuite repris dans le cadre de ce qui fut nommé un temps « géographie des représentations » pour montrer que ce courant « attachait une grande importance à ce processus qui permet d'évoquer mentalement les objets spatiaux, même si ceux-ci ne sont pas directement perceptibles ». (Bailly, 1991, p. 153). L'axe de recherche de cette géographie repose sur l'idée de la relativité de la connaissance : l'espace des humains forme une construction mentale et, par conséquent, une représentation spatiale. Les travaux analysent le rapport entre les caractéristiques structurelles et fonctionnelles d'un espace et ses représentations mentales fondées essentiellement sur le visuel. La méthode des cartes mentales pour accéder à ces dimensions et les analyser se développe (Bailly, 1991; Calenge, Lussault et Pagand, 1997). En parallèle, le laboratoire du $\mathrm{CRESSON}^{3}$ conduit des réflexions très approfondies sur le sens auditif et spécifiquement sur les effets sonores ${ }^{4}$ (Augoyard, 1992; Augoyard et Torgue, 1995) : "En résumé, souvent mesurable, très souvent lié aux caractères physiques du lieu, l'effet sonore n'est pas réductible ni à une donnée exclusivement objective, ni à une donnée exclusivement subjective. Il semble recouvrir avec pertinence cette interaction que nous cherchions à saisir entre l'environnement sonore physique, le milieu sonore d'une communauté socioculturelle et le "paysage sonore interne" à chaque individu. »

Ces deux géographies apportent les bases essentielles pour ce travail. Cependant certaines questions restent irrésolues. Comment faire le lien entre la géographie inspirée de la phénoménologie et celle des représentations? Comment combler un vide théorique dû à une double négation, celle du vécu sensoriel dans la notion de représentation de l'espace pour la « géographie des représentations », celle des avancées en neurosciences pour la géographie inspirée de la phénoménologie? Comment rendre compte de l'aspect sensible - autre que le visuel et l'auditif - et de la diversité de notre rapport aux lieux? Pourquoi l'olfactif n'est-il que très rarement pris en compte? Pour entrer dans cette complexité, un dialogue avec les neurosciences a été

\footnotetext{
3. Centre de Recherche sur l'Espace Sonore et l'Environnement Urbain UMR CNRS 1563.

4. Les recherches du CRESSON se sont élargies à la fin des années 1990 à la thématique des ambiances (Amphoux, 1998, 2004; Thibault, Grosjean, 2001 ; Thomas, 2010) et également à celles des ambiances olfactives (Balez, 2001)
} 
engagé afin de prendre connaissance des derniers modèles de la perception ${ }^{5}$ et de les mettre en dialogue avec les savoirs géographiques. Le concept de représentation des espaces s'en est trouvé enrichi. Il s'est révélé être mental et fonctionnel, comme établi par la géographie des représentations, mais également multisensoriel, émotionnel et hédonique. La complexité du processus de perception a été réaffirmée. Ce phénomène lie la création d'images multisensorielles à l'émergence d'émotion, l'excitation du centre du plaisir par un jeu de boomerang avec la mémoire. Contrairement aux idées véhiculées par la doxa, les sensations de plaisir et d'émotion se révèlent deux phénomènes neurologiques distincts. Le plaisir provient du contexte physiologique (véhiculé par les sens jusqu'au centre du plaisir), l'émotion est provoquée par le contexte psychologique.

\section{LE QUAI DE RER B À LA STATION Châ̂TElet-Les Halles de LA CHERCHEURE : ENTRE RECHERCHES DOCUMENTAIRES ET RESSENTIS}

L'objectif de cette partie est de donner une première idée de la perception du lieu à partir de l'itinéraire de la chercheure en termes de recherche documentaire, rencontres et ressentis à l'image des descriptions de Vidal de la Blache qui apportent un ensemble d'informations géographiques, historiques et techniques. En quoi, cette image mentale, fonctionnelle et multisensorielle peut-elle être généralisable?

Construite en 1977 et non rénovée jusqu'alors, la station Châtelet-Les Halles ${ }^{6}$ forme le plus grand espace de connexion de transports en commun français avec ses 750000 voyageurs journaliers en 2003, chiffre en constante augmentation (RATP, 2003). Elle forme un modèle de prouesse technique, en raison des nombreuses contraintes auxquelles les ingénieurs ont dû faire face. Elle a été édifiée au fond du «trou » des Halles à vingt-cinq mètres de

\footnotetext{
5. S'appuyant sur les ouvrages fondateurs de neurophysiologie (Changeux, 1983; Berthoz, 2003,) ou spécifiques comme L'Éloge de l'Olfaction (Holley, 1999), Le sentiment même de soi (Damasio, 2002), ce développement est avant tout le fruit de réflexions menées en collaboration avec le professeur Mac Leod (1977, 1987, 2004), ancien directeur du Laboratoire de Neurobiologie sensorielle de l'École Pratique des Hautes Études.

6. Cette description est le compte-rendu de rendez-vous du 26 mai 2004 et de la visite du site du 29 juin 2004 avec Marie Jorio, ingénieure-urbaniste de l'agence RATP Paris, responsable du projet de restructuration de la gare Châtelet-Les Halles dans le cadre du réaménagement des Halles mis en place par la Ville de Paris.
}

profondeur pour laisser à la Ville de Paris l'espace au-dessus. Elle a été construite comme une bulle à l'intérieur de la nappe phréatique, ce qui a nécessité la conception d'un système de drainage pour lutter contre la poussée d'Archimède qui fait pression sur l'édifice. Ce contexte complique le dispositif de ventilation car il faut aller chercher l'air de renouvellement loin en surface. Deux centrales de ventilation de huit mètres sur cinq mètres de large sont disposées à l'extérieur au niveau du sol (dans la cour de l'hôtel Novotel et au coin des rues Rambuteau et Lescot) : les prises d'air sont proches de la circulation automobile. La question se pose donc de la qualité de l'air qui est insufflée au niveau des quais. Ce système de ventilation est inséré dans les quais : l'air arrive du dessous (insufflation) par la voie et ressort par le dessus des quais (aspiration). Les lunules ${ }^{7}$ qui tiennent les structures contiennent les conduites d'air, les escalators et escaliers. L'épaisseur concédée par la Ville pour aménager la gare et le pôle de connexion est de dix mètres de haut. En dehors des épaisseurs de plafond et de plancher, cette épaisseur laisse trois mètres pour la salle des échanges au dessus et cinq mètres pour la hauteur de l'espace des quais en dessous. Cette restriction de hauteur explique le manque d'ampleur de l'espace des quais et l'absence de voûtes, en opposition avec toutes les autres gares RER et stations de métro de la capitale. L'espace des quais a d'ailleurs été conçu à l'image d'une gare SNCF pour leur disposition : un grand hall occupé par une succession de quais parallèles les uns aux autres, et non plusieurs tunnels propres à chaque destination comme dans les autres gares RER. Mais contrairement à une gare SNCF, cette gare RER n'est pas à ciel ouvert. L'aménagement des quais ressemble donc à celui d'une gare SNCF, à l'exception des lunules - qui occupent une grande partie du centre des quais - et des grands pylônes en béton - situés entre les quais - qui limitent la perspective. Il y a trois rangées de quais parallèles relativement éloignées les unes des autres. Chaque quai est cerné de deux voies dévolues à deux lignes de RER différentes. Le quai 1, qui nous intéresse, est voué à la ligne de RER B direction Saint-Rémylès-Chevreuse sur un côté, et au RER A direction Marne-la-Vallée-Chessy sur l'autre. Ce dispositif conduit à une fluidité maximale grâce à une logique

7. Colonnes creuses contenant le dispositif d'aération, des escaliers ou des escalators reliant la salle des échanges aux quais de RER. 
proche du mouvement brownien ${ }^{8} .800000$ personnes empruntent chaque jour le domaine RATP, 400000 à 500000 personnes utilisent la salle des échanges, 100000 changent de RER de quai à quai (RATP, 2001b). L'objectif est de diffuser les flux le plus souplement possible (grâce aux lunules masquant les poteaux de la structure, grâce aux surfaces uniformément courbes) et de les orienter par la signalétique (RATP, 2003). La répartition croisée des lignes sur les quais permet de répartir la circulation des voyageurs : un premier chassé croisé de voyageurs sur le même quai et une deuxième dans la salle des échanges.

L'espace du quai du RER B, direction Saint Rémy-les-Chevreuse, est rythmé par ces lunules qui contiennent et cachent les escaliers et les escalators prennant ainsi beaucoup de place. Entre les lunules du centre du quai, il y a un magasin de presse « Relay ». Des fauteuils de couleurs «bleu primaire » longent les murs des lunules. Dans les autres interstices, où les deux côtés du quai se rejoignent, sont disposés des banquettes de fauteuils - également «bleu primaire »- orthogonales au quai, des distributeurs automatiques d'en-cas et de boissons, des panneaux d'information de la RATP. Le sol, noir et sale, irrégulier à cause des différents dépôts de chewing-gum et autres matières poisseuses qu'aucun nettoyage ne parvient à enlever, colle sous les pas. Une lumière blanchâtre provient des tubes fluorescents qui sont installés perpendiculairement aux voies et assez espacés. Les murs, composés de céramiques bleu-roi, réfléchissent peu la lumière du plafond. Le fond sonore est composé par le bruit des freins à l'arrivée des trains, les messages sonores annonçant la fermeture des portes et secondairement des voix de voyageurs. Il n'y a pas de vis-à-vis proche de voyageurs, les quais d'en face sont trop éloignés. Sur un même quai, comme il y a deux voies, les usagers se tournent le dos. Vu sa disposition, contrairement au quai de métro, la foule ne peut pas être distrayante.

L'odeur de sulfure d'hydrogène de cette gare est une des origines de cette recherche. En effet, j'ai fréquenté cette station durant mes premières années d'université en faisant la navette entre les cours et une ville de banlieue sur la ligne B direction Saint-

8. Les voyageurs effectuent des mouvements incessants et aléatoires d'une manière indépendante les uns des autres qui conduisent à une circulation fluide.
Rémy-lès-Chevreuse. L'attente sur ce quai provoquait ${ }^{9}$ alors un ressenti d'écœurement. Son odeur me rappelait les toilettes des filles du collège qui étaient fréquemment empuantis par des «boules puantes » à l'odeur bien caractéristique d'« œuf pourri » et, par extension, les « années collége ». N'ayant pas très bien vécue cette période, cette réminiscence du collège attachée à cette odeur me plongeait dans un certain mal-être. Construire une étude à partir de ce terrain me permettait de mettre à l'épreuve l'a priori bien personnel selon lequel les usagers sur ce quai se trouvent majoritairement dans un ressenti désagréable.

Cet $a$ priori est de plus renforcé par le fait que cet espace-temps s'insère dans le mode de vie décrié en mai 1968, exprimé par le slogan «métro, boulot, dodo » et dénoncé par les situationnistes (Debord, 1996; Lefebvre, 1958). Cette odeur a également alimenté les polémiques déjà nombreuses sur cet aménagement : «La puanteur soudaine qui annonce aux voyageurs du métro l'approche de la station des Halles, c'est-à-dire de ce que des ânes ont baptisé Forum, dans l'ignorance de ce qu'est un Forum et de ce qu'il évoque [...]. Le prétendu Forum mériterait bien mieux d'être qualifié de trou : avec toutes les odeurs qui s'échappent des trous, de tous les trous, y compris de celui que Rabelais appelle tout crûment par son nom : le "trou merdeux", et auquel me fait penser ce propos désabusé de visiteurs dudit Forum et de Beaubourg : que c'est merdique » (Chevalier, 1985). Après quelques essais de désodorisation peu probants, la RATP décide d'approfondir scientifiquement les connaissances dans le domaine des odeurs en 1993. Le métro parisien devient alors « un terrain d'expérimentation sur les nuisances olfactives » (RATP, 2001a). Les stations de métro et de RER ont des odeurs récurrentes dont les origines sont diverses : absence de toilette, manque d'aération, freinage des trains (usure des patins de freinage en bois), huiles des escaliers mécaniques, entassement humain, réaction de la roche aux stations Châtelet-Les Halles, gare du Nord et Auber ainsi qu’à Madeleine. Ces stations ont en effet été creusées dans le gypse et la réduction de cette roche par l'humidité et le gaz carbonique rejetés par les voyageurs produit du sulfure d'hydrogène reconnu

\footnotetext{
9. J'emploie le passé car la fréquentation de ce quai ne m'apporte plus que légèrement cette sensation puisque les souvenirs de moments savoureux d'enquêtes atténuent le souvenir du collège et de ses « boules puantes ».
} 
communément comme « l'odeur d'œuf pourri ${ }^{10}$ ». La station métro-RER Châtelet-Les Halles est retenue pour les premières études commencées fin 1994. « La première phase du programme a consisté en une analyse olfactométrique réalisée par un panel d'expert du CEA. Elle fut renforcée par une étude psychosociologique du ressenti des voyageurs et complétée par une analyse physicochimique des composés odorants »(RATP, 2001a). À la suite de ce travail, la RATP décide d'installer de nouveaux dispositifs d'aération que ceux déjà existants et de mettre en place des procédés de désodorisation. Pour l'hydrogène sulfuré, l'installation de charbon actif en toile est à l'étude pour les stations de la ligne 14 «Pyramides » et « Madeleine ». Une méthode de photocatalyse hétérogène a été choisie pour les composés volatiles $(\mathrm{COV})$. Cette méthode repose sur la création de radicaux oxygénés attaquant les composés organiques absorbés. Ce dispositif devrait être disposé dans les billetteries. Une autre solution adoptée en 1999 est l'injection du parfum Madeleine dans les cires appliquées sur les sols après nettoyage. Cette fragrance, nommée ainsi non pas en référence à la « madeleine » de Proust mais à la station «Madeleine » de la ligne 14, créé par la société Quest, est censée sentir le muguet.

Les Halles, où se situe en souterrain la station, n'ont pas cessé d'être l'objet d'attention de la part des hygiénistes et de leurs précurseurs en raison de ses odeurs. Dès la fin du XVII ${ }^{\mathrm{e}}$ siècle, elles forment le premier terrain d'application des principes de la lutte contre les « relents morbidiques » à l'époque où elles accueillaient encore le cimetière des Innocents. Celui-ci est utilisé au moins depuis le Haut Moyen-Âge, peut-être dès le Bas-Empire (Lombard-Jourdan, 1985). Ce cimetière « reçoit durant six siècles les trépassés de vingt paroisses parisiennes » (Tabeaud, 2002), ceux de l'HôtelDieu situé non loin, les cadavres de pestiférés, des inconnus trouvés morts sur la voie publique formant ainsi le plus grand des cimetières parisiens. À partir de 1186 , le cimetière est entouré d'un mur de trois mètres de haut afin de l'isoler de la Halle. Il est doublé et surélevé d'une galerie sous arcade où l'on entasse les ossements qu'on retire des fosses communes. Les inhumations ont lieu dans une vaste

10. Il y a deux temps dans la réaction chimique : 1 . So $4 \mathrm{Ca} \rightarrow \mathrm{Sca}+2 \mathrm{O} 2$ (opération effectuée par des bactéries sulfatoréductrices)2. SCa + CO2 $+\mathrm{H} 20 \rightarrow \mathrm{CaCO} 3+\mathrm{H} 2 \mathrm{~S}$ fosse commune pouvant enterrer 1500 corps superposés. Quand elle est pleine, on en creuse une autre à côté. « Malgré le voisinage des fosses ouvertes d'où s'exhalait une odeur pestilentielle (augmentée par cela des matières fécales jetées par les habitants), malgré des charrois incessants de cadavres, malgré les charniers d'ossements, ses fresque macabres, ses épitaphes et ses tombeaux, le cimetière des Innocents fut un lieu très recherché par les oisifs, les curieux, les badauds, les polissons et aussi les entremetteuses et les fripons » (Lombard-Jourdan). À partir du XvII ${ }^{e}$ siècle, la proximité du cimetière et de la nourriture révolte de nombreux savants car s'y exhalent, selon Thion de la Chaume cité par Barles, des «émanations fétides et volatiles » (1999). Leur mobilisation conduit à la décision de fermer ce cimetière en 1785 et de le substituer par une place avec portiques, destinée également à un marché. «Pendant six mois, sous la direction du médecin Michel Thouret, "sur une étendue de deux mille toises carrées; plus de quatre-vingts caveaux funéraires ont été ouverts et fouillés : quarante à cinquante des fosses communes ont été creusées, à huit et dix pieds de profondeur, quelques-unes jusqu'au fond; et plus de quinze à vingt mille cadavres, appartenant à toutes sortes d'époques, ont été exhumés avec leur bières", sur les 90000 corps que contient le cimetière, d'après Thouret » (Barles, 1999, p. 53-54). Les ossements sont transférés dans le cimetière de la Tombe-Issoire, les actuelles catacombes. Les relents morbidiques disparaissent. Les hygiénistes inaugurent par cet assainissement du sol et le rejet des cimetières hors des murs de la capitale l'une de leurs premières actions d'importance sur l'espace urbain : "Rapidement, le désir apparaît à l'intérieur du milieu scientifique (médecins, physiciens et chimistes que l'on commence à consulter avec Turgot), non seulement d'informer la police, mais de conduire eux-mêmes une police de l'espace dont la visée serait un ordre : la santé des populations et l'espace urbain en tant qu'il a sur elle un effet durable et décisif » (Fortier, 1975). Le cas du cimetière des Innocents conduit à la formulation et à la concrétisation des premières théories pré-hygiénistes. Il établit la nécessité d'un partage entre le sain et le morbide et matérialise l'émergence d'une nouvelle autorité agissant sur la ville, celle des médecins. De ce passé odorant et de ses anciennes origines, reste une mémoire collective du lieu bien 
illustrée par Maspero dans les passagers du RoissyExpress (1990):

« [L'] arôme organique flotte en permanence dans la station Châtelet-Les Halles. [...] Anaïk, et avec elle les millions de gens qui passent par là, persiste à croire que ce sont les égouts. L'une des maximes élémentaires du voyageur étant de se méfier des explications qui vous sautent au nez, François, qui fait toujours dans le culturel suggère que cela vient de loin, de la station Auber, par le tunnel : c'est le fantôme de l'Opéra qui se promène en barque sur le lac souterrain de la Grange Batelière en agitant de ses rames les boues perstilentielles. »

À l'architecture contrainte par les impératifs techniques, au passage obligé de 800000 Franciliens par jours, aux odeurs, s'ajoute l'image négative du banlieusard et de sa vie liée au RER, entre « l'enfermement dans les cités et le voyage sans espoir à Chatelet-Les Halles » (Augé, 2008) comme l'évoque la chanson " Châtelet-Les Halles » de Florent Pagny (2000) :

\section{« Entre gris et graffitis \\ $[\ldots]$ \\ Où déambule l'ennui \\ Et se traînent des destins \\ le samedi après-midi \\ Prendre des souterrains \\ Aller voir où ça vit. »}

L'intérêt de travailler sur ce terrain est renforcé par l'enjeu urbain qu'il constitue, confirmé par le projet du réaménagement de l'espace des Halles et accentué par la difficulté de mettre ce réaménagement en place ${ }^{11}$.

À partir de documents et du ressenti de la chercheure, nous obtenons une image multisensorielle du quai de RER B dépendante de la sensibilité de l'auteure. Passant de l'échelle de l'individu à celle de l'ensemble des usagers, se pose alors la question du vécu olfactif de ceux-ci : quelles sont les odeurs identifiées? Comment sont-elles évaluées? Que peuvent-elles nous dire sur la manière dont les voyageurs vivent ce temps d'attente? En quoi cette

11. Le dispositif de concertation a souffert au départ d'incohérences et d'une faible volonté politique manquant de conduire le projet dans une impasse (Grésillon, Piault, 2004). enquête remet-elle en question le rendu sensible préalable effectué par la chercheure?

\section{DES QUESTIONNAIRES, DES ODEURS ET DES RESSENTIS}

En parallèle de cette recherche documentaire et de ce relevé de ressenti de la chercheure, il a été mis en place des enquêtes sur le quai de RER B direction Saint-Rémy-lès-Chevreuse de la station Châtelet-Les Halles pour évaluer la sensibilité olfactive des voyageurs et leur vécu. Il a été choisi de poser aux usagers uniquement trois questions pour optimiser les chances de réponse, faire en sorte que sa longueur ne soit pas un argument de refus. Puisque l'espace du quai est un lieu de traverse, il n'est fréquenté en général que le temps de l'attente d'une rame. Il faut donc convaincre et interroger les personnes entre le moment où elles arrivent sur le quai et celui où elles prennent leur train. La première question est : «Est-ce que ça sent? » Si la réponse est positive, la question qui suit est «Qu'est-ce que ça sent? ». "Qu'est-ce que ça vous évoque? » constitue la dernière interrogation. Cette dernière question permet de contourner la difficulté de nommer les odeurs grâce au moyen de l'évocation, à l'image du National Geographic Smell Survey ${ }^{12}$. Les informations concernant l'âge et la fréquence d'usage du quai terminent le questionnaire. Afin d'avoir des résultats représentatifs des usagers, l'échantillon construit sur le terrain fut proche de la composition de la population de son ensemble. Il a donc comporté $60 \%$ d'hommes, $40 \%$ de femmes, $30 \%$ de 15-24 ans, $30 \%$ de 25-34 ans, $30 \%$ de $35-54$ ans et $10 \%$ de 55 ans et plus, $20 \%$ de voyageurs occasionnels (moins d'une fois par mois), $80 \%$ de voyageurs réguliers (plus d'une fois par mois). Les moments d'enquête ont été régulièrement répartis en fonction des saisons et en fonction de la fréquentation pour avoir toute la palette des environnements possibles et pour pouvoir évaluer l'influence des facteurs de température et de foule sur le sentir. Sur les 109 questionnaires, onze questionnaires ont été ôtés de l'analyse en raison de la nature exceptionnelle des réponses obtenues le jeudi 11 septembre 2001 (dix questionnaires) et de réponses d'ordre pathologique (un question-

12. Dont une des questions était : « did the odor evoke a vivid memory? » (Wysocki, 1991, op. cit., p.288). 
naire). 98 questionnaires ont donc été analysés. Huit d'entre eux ont été très courts puisqu'à la première réponse, les enquêtés, trois femmes et cinq hommes, ont affirmé qu'ils ne sentaient rien.

À la question "Qu'est-ce que ça sent? ", les usagers ont donnés des réponses très distinctes (57 odeurs différentes sur 99 questionnaires utilisés). Seules 19 odeurs sont partagées. Dans un ordre décroissant (Illustration), ont été évoquées : la «pisse » (11 réponses), un «mélange d'odeurs » (8 réponses), les égouts ( 7 réponses), la chaleur (6 réponses), le soufre (6 réponses), la transpiration (6 réponses), les déchets (6 réponses), la saleté (5 réponses), le parfum ( 5 réponses), la pourriture ( 5 réponses), le tabac (4 réponses), la poussière (4 réponses), l'humidité (3 réponses), le «cramé » (3 réponses), le renfermé (3 réponses), les gens (2 réponses), les machines (2 réponses), le caoutchouc (2 réponses) et les excréments (2 réponses). Il s'est donc exprimé une très grande variabilité dans le sentir. Un fait étonnant reste que le parfum Madeleine à la fragrance de muguet, déposé sur le sol pour odoriser agréablement le domaine de la RATP depuis 1999 n’est jamais énoncé par les usagers. Ils ne le sentent pas.

Les analogies que 28 usagers (sur 99) font entre cet endroit et d'autres sont porteuses d'enseignement. En dehors du métro, du RER ou d'une autre station (sept enquêtés), l'odeur leur fait penser à une pièce manquant d'air ou d'aération ( 8 enquêtés) : grotte, mine, cave, « lieu enfermé comme un bar ou une salle des fêtes », la maison au retour d'un mois de vacances, à un local poubelle ou vide-ordure (4 enquêtés), aux rues pollués de Paris (3 enquêtés), à des toilettes ( 3 enquêtés), à un marais ( 1 enquêté), à l'entrée d'un vieil immeuble ( 1 enquêté), à l'espace « sous les ponts à cause des crottes de pigeons » ( 1 enquêté). Ces correspondances avec d'autres lieux mettent en valeur l'importance que revêt pour les usagers le fait que l'espace des quais soit clos. Ils montrent également le dégoût dans lequel se trouvent certains usagers (10 enquêtés), par l'évocation du local poubelle, de l'espace pollué, de toilettes, trois espaces très connotés au niveau de l'odorat. On devine une certaine peur de la contamination par les odeurs. «Version aérienne et subtile de la morale, elle est vécue comme une pénétration dans l'intimité de l'autre [...]. L'odeur est menaçante, surtout celle de l'autre, car elle imprègne l'intimité au corps défendant de l'individu qui l'inhale. Forme de possession, elle expulse de soi pour y installer un autre »(Le Breton, 2006, p. 294). Les autres représentations sont relativement neutres mais illustrent la sensation d'oppression et d'étrangeté, et également la symbolique inconsciente du lieu. Les conditions chimiques et physiques de l'environnement « quai du RER » auxquelles participent les odeurs, ce qu'elles réveillent inconsciemment, les peurs apocalyptiques liées aux attentats amènent les usagers à évaluer négativement le temps qu'ils passent sur le quai. L'évaluation hédonique du sentir sur le quai du RER B direction Saint-Rémy-lesChevreuse est majoritairement négative. L'odeur conduit à exprimer son déplaisir. La tonalité hédonique a été évoquée par 38 items différents dont les plus partagés sont : «ça pue » 5 personnes), « mauvais » (4 individus), « pas bon » (4 réponses), «indéfinissable » (4 usagers), " pas très bon » (3 personnes), «renfermé » (3 réponses) et " pas propre » (3 individus). Les évaluations hédoniques positives énoncées sont : «agréable » (2 usagers), «plaisant » (1 personne). Sur les 38 items, 23 sont de tonalité négative.

Après des analyses statistiques classiques infructueuses en raison du caractère complexe et multifactoriel de l'olfaction, le corpus des enquêtes sur le quai du RER a alors été traité par un logiciel d'analyse textuel, Alceste ${ }^{13}$. Son usage amène à la structuration des résultats sous forme de typologie d'usagers du quai du RER B selon leur sensibilité olfactive. Les usagers interrogés sur le quai du RER $\mathrm{B}$ à Châtelet-Les Halles se répartissent alors en six figures que l'on peut regrouper en deux types de sensibilités olfactives : la première reste peu développée, l'odeur est un élément de peu d'importance, la deuxième, aiguisée au contraire, se focalise sur les mauvaises odeurs.

Pour le premier type de sensorialité olfactive, il s'agit en majorité d'hommes. Ils identifient très peu les odeurs d'une manière qualitative. La caractéri-

13. Ce logiciel produit un degré « zéro » d'analyse fondé sur une statistique simple de co-occurrence de mots qui permet de rapprocher un certain nombre d'individus qui emploient le même groupe de mots dans leur phrase. L'intérêt de ce logiciel est qu'il conduit à la création de « figure » regroupant des individus en fonction de variables et à tester la validité des variables retenues et de leurs combinaisons. Il permet également de révéler une certaine complexité car il met en valeur, pour chaque figure, les variables significatives ainsi que leurs combinaisons. De plus, des extraits représentatifs des enquêtes accompagnent chaque figure permettant de l'illustrer concrètement. 
sation des odeurs est faite par analogie à un lieu ou par une sensation. "Pour moi, c'est définitivement associé au souterrain » explique un ingénieur quadragénaire. La présence odorante participe à l'identité de l'espace puisqu'elle est dite "spécifique » ou «typique». Dans ce premier type, trois figures peuvent être distinguées. Pour la première, cette contribution de l'odeur se trouve presque positive puisque les odeurs évoquées rappellent des événements qui ont pu être vécus plaisamment : les pneus brûlés des escapades d'adolescents - un technicien affirme: "Je pensais un peu au pneu qu'on faisait brûler quand on était gamin sur les grandes routes »- ou le souvenir d'une femme : "Je me souviens d'une amie à moi qui a le même parfum » raconte un homme d'affaires d'une cinquantaine d'années. La deuxième figure regroupe des hommes qui ont entre 25 et 34 ans et fréquentent le quai plusieurs fois par mois. Ils donnent aux odeurs du quai une valeur légèrement négative. Lidentification des odeurs se fait par similitude avec la sensation d'étouffement et secondairement avec l'enfance. Un étudiant en cinéma confie : "Ça m'évoque peut-être quand on est petit et qu'on s'amuse à faire comme si le lit était un tunnel pour aller jusqu'au bout du lit et quand on remonte ensuite, on a comme une petite sensation d'étouffement ", ce qui laisse à penser que l'attente sur le quai ne forme pas forcément un espace-temps de bien-être. Dans la troisième figure, des usagers qui ont entre 35 et 54 ans et qui fréquentent une fois par mois le quai, relativisent le désagrément occasionné par d'éventuelles nuisances olfactives. Un usager de longue date confie: "Je dois avoir trop l'habitude de le prendre. " Les odeurs sont identifiées grâce à l'amalgame entre l'espace des quais et les autres espaces qui l'englobent : Châtelet, Paris, ou grâce à l'analogie avec d'autres espaces : rue (bouches d'aération, pollution). L'observation d'un manque d'aération se trouve récurrente et considérée comme à l'origine des odeurs. "Ça reste un tunnel, il y a pas mal d'odeurs qui restent engoncées là dedans [...].» La fonction est évoquée comme si elle contribuait à relativiser la gêne. Les odeurs participent à l'identité de la station RER de Châtelet, de Paris et de ses rues. L'insistance sur le manque d'aération reflète une obsession proche de l'angoisse de l'étouffement mais sans la dimension dramatique. Ces interviewés ne vivent pas mal l'attente du RER dans cet espace. La référence à un lieu ou une sensation plutôt qu'à une odeur, alors que les questions appellent à une identification, illustre une olfaction peu cultivée.

Le second groupe de sensibilité olfactive observé se différencie fortement du premier par l'importance de l'identification qualitative des odeurs et par la gamme étendue des évaluations hédoniques négatives. Il est constitué de femmes en majorité et formé de trois figures. Dans la première figure, des jeunes femmes (de 15 à 24 ans), généralement interrogées l'été et qui font un usage fréquent du quai (plus d'une fois par semaine) ont un discours du domaine de la réaction «ça pue! » Les ressentis exprimés se révèlent très négatifs. «Ça pue la merde, ça dépend des fois mais quand il y a du monde, ça pue! Je ne sais pas mais ça varie entre les odeurs de pisse des gens, les mecs qui sont bourrés et qui gerbent [...]. » Les odeurs senties sont celles de l'urine (ammoniaque et hormones selon l'ancienneté du jet), la poussière. Leur représentation du quai est très négative puisque ses caractéristiques odorantes provoquent l'amalgame avec les lieux d'aisances : «Ça me fait penser aux toilettes bien sûr! » La fréquentation du quai paraît être un moment émotionnellement difficile. La seconde figure est composée des personnes ayant plus de 55 ans, fréquentant quotidiennement le quai et interrogées durant les heures de pointe. Elles identifient très précisément les odeurs. Elles prennent en compte la majorité des composés odorants pouvant être présents sur les quais : le soufré (œuf pourri, soufre), l'ammoniaquée (égouts), le moisi, les phéromones ${ }^{14}$ (la transpiration). «Oui, ça sent. Ça sent l'œuf pourri, le caoutchouc chaud [...], les égoûts, les bouches d'aération et puis le rail » explique une employée de grand magasin. La présence dans le lieu est désagréable (mise en valeur par le ressenti « désagréable » et la nature des autres perceptions exprimées par exemple ainsi : «pas frais »), gêne qui peut être renforcée par le motif de fréquentation de la station (aller au travail). Mais cette gêne n'empêche pas une réelle curiosité pour la dimension odorante de la station. La précision des descriptions met en évidence une grande sensibilité olfactive de ces individus. Pour certains, cela s'explique par leurs professions (chimistes, ouvriers en métallurgie). Le vécu sur le quai n'est pas totalement négatif puisqu'il reste propice à l'épanouissement d'une certaine curiosité. Les femmes âgées

14. «Signal chimique de communication [...].» (Holley, 2006, p. 247). 
de plus de 55 ans, constituant la troisisème figure, donnent au quai une odeur de cave avec une évaluation hédonique faiblement négative. Les odeurs se caractérisent par leur origine, particulièrement le manque d'aération, et secondairement la saleté, le monde. "C'est désagréable, ça vient des gens, de la promiscuité, le manque d'aération, le manque d'air [...]. » L'odeur d'hydrogène sulfurée (l'œuf pourri) est identifiée. Une institutrice à la retraite affirme : "J'ai déjà senti ça. Une cave, un lieu de renfermé et de chaud en même temps [...]. » La cave possède des situations souterraines banales, l'analogie suppose que le contexte sensoriel du quai a les attributs habituels des sous-sols. La cave est attachée à l'idée de maison, espace de refuge mais également au lieu des morts, voire à la localisation de l'enfer. Par ailleurs, la cave est associée aussi à l'immeuble, qui dans les « grands ensembles » n'est pas un espace positif ni même neutre (trafics en tout genre, espace de non-droit). La représentation de cet espace se révèle soit légérement positive, soit légérement négative. Il est difficile d'aller plus en avant dans l'étude de cette analogie car en raison de la briéveté du questionnaire, on ne connait pas le type d'habitat des personnes rencontrées.

\section{GrÂCE À CETTE ENQUÊTE ET À L'ÉCLAIRAGE DES NEUROSCIENCES, UNE COMPRÉHENSION DES DECRIPTIONS « SÈCHES » DES LIEUX}

Les questionnaires des usagers du quai du RER mettent en valeur une grande diversité dans la perception des odeurs, dans leur évaluation et dans l'acuité de l'olfaction entre les individus. Nous observons donc que décrire les odeurs d'un lieu demande à mettre en place une méthode d'enquête pour recueillir l'ensemble de la palette des différentes odeurs senties et des différents ressentis. Cet exemple du quai de RER B direction Saint-Rémylès-Chevreuse le met bien en valeur : la chercheure peut se considérer comme un «observateur standard ${ }^{15} »$ et généraliser son ressenti pour ce qui est

15. La vision, l'audition et en partie la somesthésie - anciennement le toucher - possèdent des capteurs physiques comme nous l'avons déjà évoqué. Leur fonctionnement est pratiquement identique d'une personne à l'autre. Pour ces sens physiques, il est donc possible de considérer l'existence d'un « observateur standard » (concept psychophysique proposé par les chercheurs anglo-saxons dès le XIX ${ }^{e}$ siècle) dont nous pouvons de la description des perceptions visuelles et auditives venant des récepteurs physiques. Elle dispose - de plus - pour étayer ses propos de nombreux documents techniques et historiques. Par contre, lorsqu'on compare son ressenti olfactif (partie 2) et celles des usagers (partie 3), il n'est ni méthodologiquement ni déontologiement possible de procéder de même, de prendre son «sentir » comme modèle, tant l'identification des odeurs et ses ressentis se révèlent bien personnels.

Cette particularité est liée à la nature du sens olfactif. Sa spécificité première provient de son polymorphisme génétique ${ }^{16}$ qui implique, avec sa quasi-impossibilité d'une catégorisation commune, une grande pauvreté du référentiel commun ${ }^{17}$ concernant les odeurs. En effet, alors que quelques gènes du génome humain codent l'audition et la vision, 347 codent les récepteurs olfactifs. De plus, $50 \%$ de ces gènes sont polymorphes ${ }^{18}$ c'est-à-dire que chacun d'eux peut exister sous des formes différentes. De ce fait, deux personnes ayant les mêmes gènes peuvent posséder des récepteurs olfactifs distincts. Un simple calcul de probabilité montre que ce caractère polymorphe des gènes codant l'olfaction rend pratiquement impossible le fait que deux observateurs perçoivent une seule et unique odeur en présence d'un même stimulus (composé d'une ou de plusieurs molécules). Cette particularité induit qu'en matière d'identification de l'odeur, il y ait peu de référentiel collectif (Holley, 2007). Cela induit également le fait que nous décrivons une odeur non pas par sa qualité mais par sa source supposée ${ }^{19}$ : on

généraliser les perceptions. Les chimiorécepteurs de l'olfaction, du goût et d'une partie de la somesthésie ont au contraire un fonctionnement très significativement différent d'une personne à l'autre. L'« observateur standard » n'existe pas pour ces sens.

16. « La complexité et la variabilité d'un phénotype qui ne compte pas moins de 347 gènes pour coder nos récepteurs olfactifs [...] font que les odeurs [...] forment un continuum hautement multidimensionnel et que la seule classification possible concerne les observateurs que nous sommes et non les observés odorants [...]. Il nous faut accepter et jeter les bases nouvelles qui intègrent le fait qu' [...] une odeur [est] exactement autant déterminé[e] par les protéines réceptrices du sujet qui sent que par les molécules de l'objet qui est senti » (Mac Leod, 2004c).

17. Il y a $17 \%$ de consensus sur l'intensité, l'identification et l'appréciation pour une même formule chimique odorante d'après la thèse de Jérôme Jallat (1995 - ENGREF).

18. «En génétique, désigne le fait qu'une région étroitement définie d'un chromosome, ou même un seul gène, détermine plusieurs traits phénotypiques, plusieurs manifestations comportementales ou physiologiques dans un population donnée d'individus. » (Holley, 2006, p. 247).

19. «On reconnaît une odeur plus facilement qu'on ne la décrit : aucune des références spatiales qui s'appliquent aux autres sensations ne lui convient. Seule, la source d'une odeur est véritablement appréhendée comme objet, à telle enseigne que nous ne savons nommer celle-ci que d'après celle-là. » (Holley, Mac Leod, 1977, p. 729). 
dit «ça sent les toilettes », par une évaluation hédonique : «ça pue!» ou encore par le recours à un souvenir autobiographique : «Ça me rappelle notre maison de vacances quand on arrivait [...], quand j'étais petite $[\ldots]$ » comme nous avons pu l'entendre sur le quai du RER. Cette différence est manifeste si on compare l'olfaction à la perception visuelle. On dit c'est «bleu », non «c'est la couleur de la mer». Par manque de vocabulaire, le cerveau fait donc appel à la mémoire autobiographique pour l'identification de l'odeur. Si celle-ci a déjà été sentie, le souvenir et le contexte sensoriel du premier sentir s'imposent à la conscience et permettent indirectement l'identification. Puisqu'elle est liée au plaisir et à l'émotion du primo-souvenir (Sevelinges et al., 2008, 2009), l'évaluation hédonique d'une odeur comporte d'énormes variations interpersonnelles : chaque individu conçoit des références liées à ses expériences intimes. Prendre en compte et rendre compte du sensible en ce qui concerne l'olfactif se révèle alors lancer un réel défi.

Cela nous éclaire sur les choix de nos illustres pairs de n'intégrer à l'excercice de la description que le visuel. On peut tout de même regretter, comme Sion (1934), leur excès de zèle. En effet, l'épure de cet excercice fondateur jusqu'à ne garder que le strict visuel, qui oublie la perception auditive ainsi que les émotions et plaisirs y afférant, n'était pas nécessaire pour respecter son objectif d'universalisme. Car, nous l'avons en effet évoqué, pour les sens physiques, il existe un « observateur standard ».

\section{Conclusion}

Ayant choisi de construire un dialogue avec les neurosciences, il aurait été facile voire caricatural de défendre un point de vue naturaliste sur le sujet. Cet article ne le développe pas mais les conclusions de cette recherche montrent que la complexité de l'interaction entre l'être humain et les milieux urbains n'a été qu'effleurée (Grésillon, 2010a, 2010b). Le sentir et le ressentir sur le quai de RER à Chateletles-Halles dépend des données propres à l'usager - ce que Morin appelle « les dimensions trinitaires de l'homme » (2008) - c'est-à-dire sa physiologie (âge, sexe, état de santé), de sa psychologie (de son histoire personnelle), de son appartenance sociale et de son origine culturelle, et également des caractéristiques physico-chimiques et sociale du lieu. Mais quelles sont les parts respectives de chacun de ses variables humaines? Sur cette question, au vue de sa complexité, le champ des recherches reste infini.

\section{Bibliographie}

Amphoux P. (dir.), 1998. La notion d'ambiance, une mutation de la pensée urbaine et de la pratique architecturale, Paris, Plan Urbanisme Construction Architecture (PUCA), ministère de l'Équipement, des Transports et du Logement.2013-0705T1 1:56:00

La bibliographie n'est pas aux normes. Cf : consignes aux auteurs. Merci d'avance de reprendre et de corriger les réf une à une.

Amphoux P., Thibaud J.-P., Chelkoff G. (dir.), 2004. Am-

biances en Débats, Grenoble, Éditions À la Croisée.

Augé M., 2008. Le Métro revisité, Paris, Seuil, La Librairie du $\mathrm{XXI}^{\mathrm{e}}$ siècle.

Augoyard J.-F. (dir.), 1992. La qualité sonore des espaces habités./ Sonic quality in the Living Environement, Paris/Grenoble, CRESSON/Ministère de la Recherche.

Augoyard J.-F., Torgue H., 1995. À l'écoute de l'environnement. Répertoire interdisciplinaire des effets sonores. Marseille, Editions Parenthèses.

Bailly A., 1974. La Perception des paysages urbains - essai méthodologique, Espace Géographique, III (3), p. 211-217.

Bailly A., 1981. La Géographie du bien-être, Paris, PUF.

BALEz S., 2001. Ambiances olfactives dans l'espace construit, Thèse de doctorat d'architecture.

Barles S., 1999. La Ville délétère, Médecins et ingénieurs dans l'espace urbain, XVIII ${ }^{e}$-XIX ${ }^{e}$ siècle, Paris, Champ Vallon, coll. « milieux».

Berque A., 1986. Médiance de milieux en paysages, Paris, Belin/ Reclus Géographiques.

Berthoz A., 2003. La Décision, Paris, Odile Jacob.

Calenge C., Lussault M., Pagand B., 1997. Figures de l'Urbain, des villes, des banlienes et de leurs représentations, Tours, collection «Sciences de la ville », $\mathrm{n}^{\circ} 14$, Publication de la Maison des Sciences de la Ville Université FrançoisRabelais.

Changeux J.-P., 1983. L'Homme Neuronal, Paris, Fayard, collection « Pluriel ».

Chelkoff G., Thibaud J.-P. et al., 1997. Ambiances sous la ville. Une approche écologique des espaces publics souterrains. CRESSON/Plan Urbain.

Chevalier L., 1985. Les Parisiens, $2^{\mathrm{e}}$ édition, Paris, Hachette Pluriel.

Damasio A.-R., 2002. Le Sentiment même de soi-corps, émotions, conscience, Paris, Odile Jacob, $2^{\mathrm{e}}$ édition.

Dardel E., 1990. L'Homme et la Terre. Nature de la réalité géographique, $1^{\text {re }}$ édition 1952, Paris, CTHS.

Debord G., 1996. La Société du spectacle, Paris, Gallimard.

Fallet R., 1977. Les Halles, la fin d'une fête, Paris, Duculot, p. 1-2. 
Feildel B., 2010. Espaces et projets à l'éprewve des affects. Pour une reconnaissance $d u$ rapport affectif à l'espace dans les pratiques d'aménagement et d'urbanisme, Doctorat d'urbanisme, École Polytechnique de l'université de Tours, Tours, université F.-Rabelais.

Fortier B., 1975. La Politique de l'espace parisien, Paris, Corda. Frémont A., 1976. La Région, espace vécu, Paris, PUF.

Grésillon L., 1997. Le Paris qui sent: les odeurs du quartier de la Huchette, mémoire de Maîtrise sous la direction de Jean-Robert Pitte, université Paris Sorbonne.

Grésillon L., 1998. Le Paris qui sent : les odeurs du quartier de la Huchette, dans Dulau, R., Pitte, J.-R., La Géographie des Odeurs, Paris, L'Harmattan, p. 179-207.

GréSILlon L., 2010a. Les odeurs du quai de RER à Châteletles Halles : pour une approche sensorielle du bien-être en ville, Nature Sciences et Société, 2/2010, vol. 18, p. 122-132.

Grésillon L., 2010b. Sentir Paris, Bien-être et matérialité des lieux, Paris, QUAE, «Indisciplines ».

Grésillon L., Piault F., 2004. « Sortir du piège », Le Monde, 13 novembre 2004.

Grosjean M., Thibaud J.-P. (dir.), 2001. L'espace urbain en méthodes, Marseille, Parenthèses.

Holley A., 2006. Le Cerveau gourmand, Paris, Odile Jacob.

Holley A., 1999. L'Eloge de l'olfaction, Paris, Odile Jacob.

Holley A., 2007. Les secrets de l'odorat, Cerveau et Psycho, $\mathrm{n}^{\circ} 21$, p. 50-55.

Holley A., Mac Leod, P., 1977. Transduction et codage des informations olfactives chez les vertébrés, Journal de Physiologie, t. 73, p. 725-828.

Hoyaux, A.-F., 2002. «Entre construction territoriale et constitution ontologique de l'habitant : Introduction épistémologique aux apports de la phénoménologie au concept d'habiter », Cybergeo : European Journal of Geography [En ligne], Epistémologie, Histoire de la Géographie, Didactique, article 216, mis en ligne le 29 mai 2002, consulté le 28 février 2012. URL : [http://cybergeo.revues.org/1824; DOI : 10.4000/cybergeo.1824].

JaLlat J., 1995. Mise au point d'une méthode sensorielle d'analyse descriptive des odeurs en parfumerie, Thèse de l'ENGREF.

Jorio M., 2005. « Un pôle de transport à ouvrir vers la ville », Urbanisme, janv.-fév. 2005, n³ 340, p. 20-22.

Le Breton D., 2006. La Saveur du monde, Une anthropologie des sens, Paris, éditions Métailié.

Lefebvre H., 1958. Critiques de la vie quotidienne, t. 1, Introduction, $2^{\mathrm{e}}$ édition, Paris, L'Arche.

LÉvy J., Lussault M., 2003. Dictionnaire de la géographie et de l'espace des sociétés, Paris, Belin.

Lombard-Jourdan A., 1985. Aux origines de Paris, La genèse de la Rive droite jusqu'en 1223, $1^{\text {re }}$ édition 1976, Paris, CNRS, p. 53-59.

Mac Leod P., 1987. Si on ne sent pas pareil c'est qu'on nez pas pareil, dans Blanc-Mouchet, J., Odeurs, l'essence d'un sens, Paris, Autrement, p. 74-79.

Mac Leod P., 2004a. Communication personnelle.

MAC LEOD P., 2004b. Enfin un podium pour l'olfaction!, Médecine/sciences, $\mathrm{n}^{\circ} 2$.
Mac Leod P., 2004c. Les neurosciences aujourd'hui : goût, conscience, plaisir, Revue des œnologues, n 111 , p. 9-10.

Manola T., 2012. Conditions et apports du paysage multisensoriel. Pour une approche sensible de l'urbain, Doctorat d'urbanisme, Université Paris-Est.

Martouzet D., 2007. Le rapport affectif à la ville : premiers résultats, dans Paquot T., Lussault M., Younès C., Habiter, le propre de l'humain : villes, territoires et philosophie, Paris, La Découverte, p. 171-191.

Martouzet D., 2010. Amour/désamour de la ville : approche individualiste. Esquisse d'une méthode générale pour l'examen du rapport affectif à la ville et à sa construction comme objet de recherche, dans Salomon Cavin J., Marchand B., Antiurbain. Origines et conséquences de l'urbaphobie, Lausanne, PPUR.

Martouzet D., Martouzet D., Bailleul H., Feildel B., GaiGNARD L., 2010. La carte : fonctionnalité transitionnelle et dépassement du récit de vie, Nature, Sciences, Sociétés, Vol. 18, n² 2, p. 158-170.

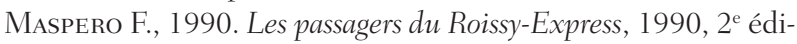
tion, Paris, Points Seuil, p. 8.

Mathieu et al., 2004. "Habiter le dedans et le dehors : la maison ou l'Eden rêvé et recréé ", Jeunes recherches. La vitalité d'un laboratoire, Strates, 11/2004, p. 267-288.

Mathieu N., 1996. Rural et urbain : unité et diversité dans l'évolution des modes d'habiter, dans Jollivet M., Eizner N. (éd.), L'Europe et ses campagnes, Paris, Presses de la FNSP, p. 187-216.

Merleau-Ponty M., 1976, Phénoménologie de la Perception, Paris, Gallimard, coll. « Tel », [1 $1^{\text {re }}$ édition 1945].

RATP, 2001a, « La RATP et le traitement des odeurs », SavoirFaire, ${ }^{\circ} 39$, p. 16-21.

RATP, 2001 b, Diagnostic urbain - Le site de Châtelet-les-Halles : Analyse des usages et recherche de la multimodalité, Paris, RATP, Département des projets.

RATP, 2003, Les Halles, gare centrale et porte de Paris - Éléments pour le réaménagement du pôle d'échange au sein du quartier, Politiques et études amonts, Version 2, Paris, RATP.

Reclus E., 1982. L'Homme et la terre, [1 ${ }^{\text {re }}$ édition 1905].

Salomon Cavin J., 2005. La ville, mal-aimée - Représentations anti-urbaines et aménagement $d u$ territoire en Suisse : analyse, comparaisons, évolution, Lausanne, PPUR.

Salomon Cavin J., Marchand B., 2010. Antiurbain. Origines et conséquences de l'urbaphobie, Lausanne, PPUR.

Sevelinges Y., Mouly A.-M., Lévy F., Ferreira G., 2009. Long-term effects of infant learning on adult conditioned odor aversion are determined by the last preweaning experience, Developmental Psychobiology, 51, 5, p. 389-398.

Sevelinges Y., Sullivan R. M., Messaoudi B., Mouly A.-M., 2008. Neonatal odor-shock conditioning alters the neural network involved in odor fear learning at adulthood, Learning \& Memory, 2008, 15, 9, p. 649-656.

Sion J., 1934. L'Art de la description chez Vidal de la Blache, Mélanges de philologie, d'histoire et de littérature offerts à Joseph Vianez, Paris, p. 479-487.

STOck M., 2004. L'habiter comme pratique des lieux géographiques, EspacesTemps.net, Textuel. 
Tabeaud M., 2002. La Mort en Île de France, Paris, Publications de la Sorbonne.

Thomas R., 2010. Marcher en ville. Faire corps, prendre corps, donner corps aux ambiances urbaines, Paris, Éd. des Archives Contemporaines.

Vidal de la Blache, 1895. Atlas Général d'histoire et de géographie, Paris, Armand Colin.

Vidal de la Blache, 1994. Tableau de la géographie de la France, $1^{\text {re }}$ édition 1908.

Wysocki C.-J., Pierce J.-D., 1991, Geographic, Cross-Cultural, and Individual Variation in Human Olfaction, Smell and Taste in Health and Disease, New York, edited by T. V. Getchell T.V. et al., Raven Press, p. 287-314.

\section{Cdrom}

Pagny F., 2000, Châtelet-Les Halles, Label Mercury.

\section{Émission de radio}

Morin E., 2008. «Les Chemins de la connaissance », France Culture, intervention du 7 janvier 2008 de 19 h 30 à 20 h 00 . 